\title{
SARS-CoV-2 cell-to-cell infection is resistant to neutralizing antibodies
}

\author{
Natalia Kruglova ${ }^{1}$, Andrei Siniavin $^{2,3}$, Vladimir Gushchin $^{2}$, and Dmitriy Mazurov ${ }^{1^{*}}$ \\ ${ }^{1}$ Center for Precision Genome Editing and Genetic Technologies for Biomedicine, Institute of Gene \\ Biology RAS, Moscow, 119334, Russia \\ ${ }^{2}$ N.F. Gamaleya National Research Center for Epidemiology and Microbiology, Ministry of Health \\ of the Russian Federation, Moscow, 123098, Russia. \\ ${ }^{3}$ Department of Molecular Neuroimmune Signalling, Shemyakin-Ovchinnikov Institute of \\ Bioorganic Chemistry, Russian Academy of Sciences, Moscow, 117997, Russia \\ * Correspondence should be addressed to D.M. (dvmazurov@yandex.ru)
}

Key words: SARS-CoV-2, cell-to-cell infection, serum neutralization, intron-containing reporter

\begin{abstract}
The COVID-19 pandemic caused by SARS-CoV-2 has posed a global threat to human lives and economics. One of the best ways to determine protection against the infection is to quantify the neutralizing activity of serum antibodies. Multiple assays have been developed to validate SARS-CoV-2 neutralization; most of them utilized lentiviral or vesicular stomatitis virus-based particles pseudotyped with the spike (S) protein, making them safe and acceptable to work with in many labs. However, these systems are only capable of measuring infection with purified particles. This study has developed a pseudoviral assay with replication-dependent reporter vectors that can accurately quantify the level of infection directly from the virus producing cell to the permissive target cell. Comparative analysis of cellfree and cell-to-cell infection revealed that the neutralizing activity of convalescent sera was more than tenfold lower in cell cocultures than in the cell-free mode of infection. As the pseudoviral system could not properly model the mechanisms of SARS-CoV-2 transmission, similar experiments were performed with replication-competent coronavirus, which detected nearly complete SARS-CoV-2 cell-to-cell infection resistance to neutralization by convalescent sera. Based on available studies, this is the first attempt to quantitatively measure SARS-CoV-2 cell-to-cell infection, for which the mechanisms are largely unknown. The findings suggest that this route of SARS-CoV-2 transmission could be of great importance for treatment and prevention of COVID-19.
\end{abstract}

\section{Importance}

Immune surveillance of viral or bacterial infections is largely mediated by neutralizing antibodies. Antibodies against the SARS-CoV-2 spike protein are produced after vaccination or infection, but their titers only partly reflect the degree of protection against infection. To identify protective antibodies, a neutralization test with replicating viruses or pseudoviruses (PVs) is required. This study developed lentiviral-based PV neutralization assays that, unlike similar systems reported earlier, enable quantitative measurement of SARS-CoV-2 neutralization in cell cocultures. Using both PVs and replication-competent virus, it was demonstrated that SARS-CoV-2 cell-to-cell infection is considerably more resistant to serum neutralization than infection with purified viral particles. The tests are easy to set up in many labs, and are believed to be more informative for monitoring SARS-CoV-2 collective immunity or entry inhibitor screening. 


\section{Introduction}

SARS-CoV-2 is a respiratory virus, a causative agent of COVID-19. The primary target of the virus is the airway epithelium of the upper respiratory tract. ${ }^{1-3}$ During the course of the disease, the virus can descend to the lower respiratory tract, infecting bronchial epithelium and type II pneumocytes. ${ }^{4}$ The main receptor for SARS-CoV-2, angiotensin-converting enzyme 2 (ACE2), ${ }^{1,5,6}$ determines the viral tropism, which is not restricted to the respiratory epithelium and in certain cases can infect enterocytes, as well as kidney, heart, brain, and other cell types. ${ }^{7,8}$ Molecules other than ACE2 have been reported to be involved in SARS-CoV-2 entry, such as neuropilin- $1,{ }^{9,10} \mathrm{AXL},{ }^{11}$ and $\mathrm{CD} 147$, although the role of the latter is speculative. ${ }^{12,13}$

SARS-CoV-2 entry is mediated by the spike (S) protein. ${ }^{14}$ The $S$ protein belongs to trimeric class I fusion proteins ${ }^{15}$ that undergo substantial conformational changes when bound to a cellular receptor, leading to fusion between viral and cell membranes. ${ }^{16,17}$ The extracellular portion of the spike consists of two subunits: S1 binds to ACE2 and S2 mediates the viral fusion. ${ }^{17}$ A newly synthesized spike exists in a metastable prefusion state. ${ }^{17}$ Following attachment to permissive cells, the receptor binding domain (RBD) in the S1 subunit transitions between the inactive 'down' position and the accessible 'up' position for interaction with ACE2. ${ }^{18-20}$ However, binding the S protein to ACE2 is not sufficient for triggering membrane fusion, because the fusion peptides of coronaviral $\mathrm{S}$ proteins have a 'hidden' localization inside the S2 subunit. ${ }^{17}$ Proteolytic cleavage at the S2' site releases fusion peptide. This process is mediated by several host proteases: TMPRSS2, and lysosomal cathepsins B and L., ${ }^{6,}$ Depending on localization within the target cells, these proteases largely determine virus entry sites; plasma membrane in the case of TMPRRS2 or endosomes when cathepsins are engaged. ${ }^{14,22}$ In this regard, SARS-CoV-2 is not unique and demonstrates features that have long been known from other coronaviruses. ${ }^{21}$ In contrast to the S2' site, the furin cleavage site at the S1/S2 boundary is a special feature of SARS-CoV-2 that generally distinguishes it from other beta-coronaviruses, such as SARS-CoV, ${ }^{23}$ with the exception of MERS-CoV, where it is present. ${ }^{21}$

An invaluable instrument for coronavirus entry inhibitor assessment is pseudoviruses. They are safe, reliable, and fast for generating quantitative data relative to fully competent viruses, which often require strict regulation when working with them. The SARS-CoV-2 spike protein is sufficient to mediate pseudovirus entry and many pseudoviral systems were developed during the COVID-19 pandemic, generally using HIV, ${ }^{6,24-28} \mathrm{MLV}^{2,29,30}$ or VSV 3,5,31,32 platforms. In comparison to retro- or lentiviral particles, which require 48 hours to get infectivity results, results for VSV-based particles can be obtained within 24 hours of infection and at higher titers, although the production is more labor-intensive. ${ }^{6,33,34}$ In general, the choice of pseudoviral system is primarily dictated by the preferences of a particular research group. ${ }^{35}$ Using pseudoviral tests, large amounts of data on the inhibitory activity of sera from convalescent and vaccinated individuals, monoclonal antibodies, proteins, peptides, and small molecules were collected and analyzed. ${ }^{25-29,36}$

Despite fast progress in SARS-CoV-2 entry inhibitor evaluation using pseudoviruses, the vast majority of developed systems are capable of measuring infectivity only with purified particles. Meanwhile, the largely unknown - and potentially important - mechanism of SARS-CoV-2 spread from cell-to-cell has not been evaluated with pseudoviruses. This study describes the $S$ protein pseudotyped lentiviral system for measuring SARS-CoV-2 infection in both cell-free and cell-to-cell infection settings. 
This was achieved with replication-dependent reporter vectors that were developed earlier. ${ }^{37,38}$ The key feature of these vectors is that the reporter is silent in the pseudovirus-producing cells, but active after infection of the target cells and completion of one cycle of viral replication. This enables infectious events to be measured directly in cocultures of producer and target cells at zero background level. The concept was effectuated by placing a reporter cassette in reverse orientation relative to HIV-1 genomic RNA and through interruption it with an intron, that prevented a functional reporter protein expression from LTR and CMV promoters in transfected (producer) cells. Comparative analysis of SARS-CoV-2 infection in two transmission settings revealed a substantially lower capacity of convalescent sera to neutralize infection in cell cocultures than in a cell-free test. This effect was reproduced with replication-competent SARSCoV-2, indicating that cell-to-cell transmission of SARS-CoV-2 and its elevated resistance to entry inhibitors are important parameters for monitoring anti-viral immunity and developing anti-coronaviral drugs.

\section{Results}

Generation and optimization of a SARS-CoV-2 pseudoviral system to measure cell-free infection.

Pseudoviruses (PVs) are viruses enveloped with a heterologous surface protein that changes their natural tropism. Unlike native systems, heterologous protein envelopes are often incorporated into PV particles at lower efficiency. A number of studies have been focused on optimizing lenti- and retroviral systems pseudotyped with the SARS-CoV S protein. Giroglou et al. showed that C-terminal truncation of the $S$ protein increased retroviral particle titers, explained by the removal of the ER retention signal and exposure of the S protein to the cell surface. ${ }^{39}$ Moore et al. found that both C-terminal truncation and substitution of the cytoplasmic portion of the $\mathrm{S}$ protein with eight amino acids from the C-terminus of HIV-1 gp41 increased the level of infectious lentiviral particle production. ${ }^{40}$ Later on, the spike protein from SARS-CoV-2 with a cytoplasmic portion deleted was used in a number of pseudoviral test systems. ${ }^{6,32,41-43}$ The furin cleavage site that was present in SARS-CoV-2 S but not in the SARS-CoV S protein is thought to be involved in spike maturation, virus entry, and syncytium formation ${ }^{5}$ and, therefore, can also affect infectivity measured with PVs.

In order to establish an HIV-based infection system, the SARS-CoV-2 S protein was modified by deleting the last 19 amino acids $(\triangle \mathrm{C} 19)$ or substituting them with eight amino acids from HIV-1 gp41 (H2). These modifications were either combined with the mutation in the furin cleavage site RRAR to $A(\Delta F)$, or left uncombined, to generate the six variants of spike protein indicated in Figure 1A. Next, a SARS-CoV-2 permissive HEK 293T cell line was established with a stable expression of the human ACE2 receptor via lentiviral transduction and FACS sorting (Figure 1B). A cell-free infectivity assay was set up, as schematically illustrated in Figure 1C. PVs were generated by co-transfecting 293T cells with one of the S protein coding plasmids, HIV-1 packaging vector pCMV-dR8-2, and an improved intron-regulated reporter vector pUCHR-inLuc-mR, capable of measuring both cell-free and cell coculture infections using the mean of luciferase activity. ${ }^{37,38}$ Additionally, the pUCHR-IR-GFP reporter plasmid without an intron was used to evaluate cell-free infectivity levels using flow cytometry. At 48 hours post-transfection, supernatants containing PVs were harvested and concentrated by centrifugation. Equal amounts of PVs were added to 293T/ACE2 cells for 48 hours, and levels of infection were estimated by measuring luciferase activity or percentage of GFP-positive cells, depending on reporter type. The resulting values of infection were 
normalized to $\mathrm{p} 24$ levels, and presented relative to the values obtained for the wild-type $\mathrm{S}$ protein. As shown in Figure 1D, $\triangle C 19$ moderately increased the level of infection, while the $\mathrm{H} 2$ modification had no or little effect on infectivity. By contrast, the $\Delta \mathrm{F}$ mutation resulted in about a 1.5 log increase in PV infectivity. On $\Delta F$ background, however, the improving effect of $\Delta C 19$ was much less pronounced than detected without $\triangle F$. PV titration was used to confirm a substantial effect of the $\Delta F$ mutation on the level of PV transduction ( 20 fold enhancement in many PV dilutions) (Figures $1 \mathrm{E}$ and $\mathrm{F}$ ). The increased infectivity of the $\triangle F$ mutant PVs was not accompanied by an increase in $\mathrm{S}$ protein expression on PVproducing 293T cells (Figure 1G). Thus, it was unclear whether $\Delta F$ infectivity was enhanced from $S$ incorporation into PVs or if this was a feature of the 293T cellular system, in which S processing by furin is important during the fusion step of the viral life cycle.

In summary, a SARS-CoV-2 cell-free infection test was developed in a 24-well plate format with a high level of sensitivity. Using the $\triangle \mathrm{F} \Delta \mathrm{C} 19$ modification of the SARS-CoV-2 S protein, 50-60\% GFP transduction and about 4 log over the background elevation of luciferase activity was achieved, making consecutive inhibitory analysis accurate and reproducible.

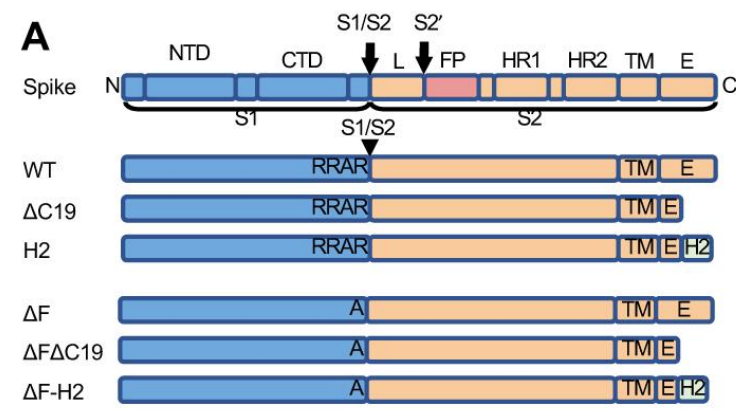

C

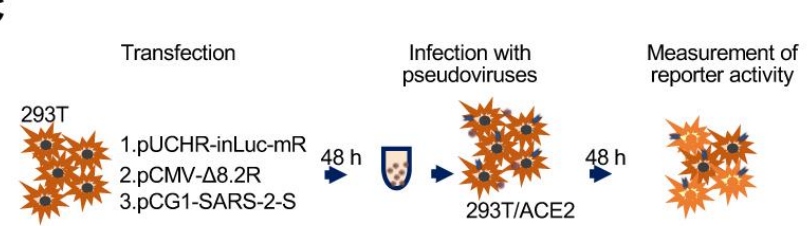

B

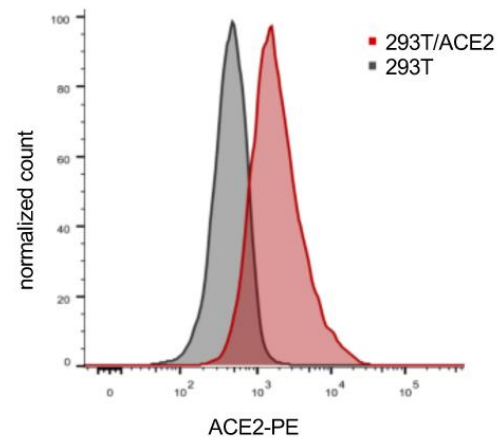

D

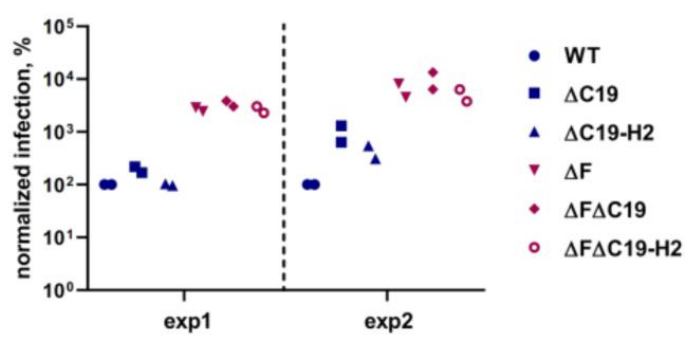

E

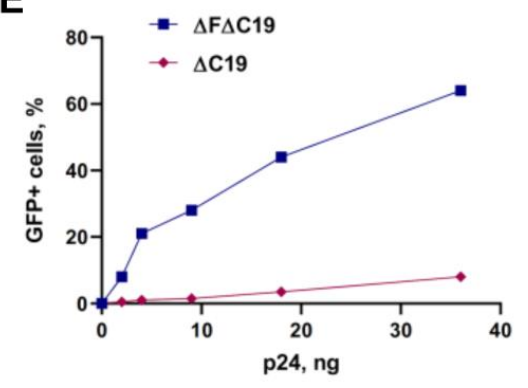

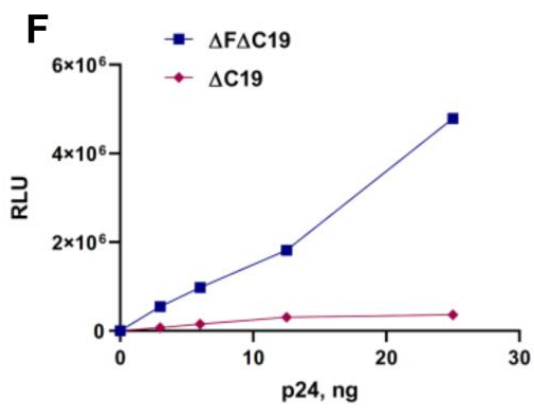

G

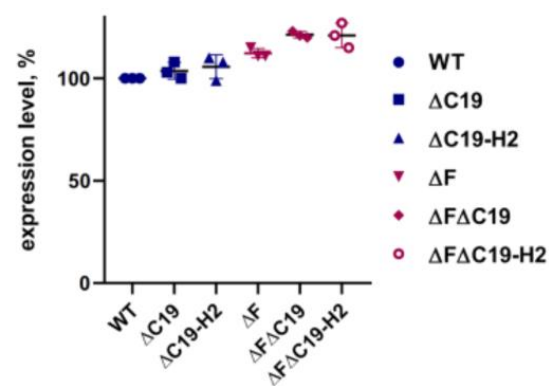

Figure 1. Development of a SARS-CoV-2 cell-free infection test with PVs.

A. A schematic illustration for S-protein variants used in pseudovirus infection tests. Six different constructs of the S protein were generated by PCR mutagenesis B. Evaluation of the ACE2 surface expression on 293T cells stably transduced with the hACE2 using flow cytometry. C. Experimental setup for SARS-CoV-2 cell-free infection measurement. D. The levels of Infection detected with different variants of the $S$ protein. PVs were added to 293T/ACE2 cells in a equal amount based on HIV-1 Gag quantification. The luciferase activity measured for a mutant spike was normalized to that obtained for the wild-type S protein. Two independent experiments with two different PV preparations were performed. E,F. The levels of cell-free infection with indicated PVs were measured using either GFP (E) or inLuc (F) reporter. G. The 
levels of S protein expression on PV producing cells estimated by flow cytometry. 293T cells were transfected to express indicated variants of protein $\mathrm{S}$ and stained with convalescent human serum in 48 hours. Median fluorescence intensity (MFI) level was calculated for the every mutant in the gate of transfected cells and normalized to the MFI detected for wild-type S protein.

\section{Development of a SARS-CoV-2 pseudoviral system to quantify cell-to-cell infection.}

In order to evaluate SARS-CoV-2 cell-to-cell infection, a one step transfection-infection assay with the inLuc-mR reporter vector described earlier was set up. ${ }^{37,38}$ Briefly, 293T/ACE2 cells were cotransfected with viral vectors, as outlined above, for cell-free infection. In approximately 12-16 hours, transfected cells started to produce PVs, which infected nearby 293T/ACE2 cells. At 48 hours posttransfection, one cycle of replication was complete and luciferase activity can be measured (Figure 2A). Using this assay, the levels of infection with one of the six variants of the $\mathrm{S}$ protein were quantified. Wild type, $\Delta \mathrm{C} 19$, and $\mathrm{H} 2$ proteins did not mediate infection at all, however, all three variants bearing the $\Delta \mathrm{F}$ mutation supported a good level of infection. The addition of $\Delta \mathrm{C} 19$ to $\Delta \mathrm{F}$ increased the level of infectivity by $0.5 \mathrm{log}$, while the $\mathrm{H} 2$ modification had no effect on the signal (Figure 2B). We have previously demonstrated that intron-regulated reporter vectors do not detect infection in cell syncytia, as the reporter protein can be expressed only in actively replicating target cells. ${ }^{37}$ Therefore, the ability of differently modified S protein variants to induce syncytia formation in 293T/ACE2 cells was examined. Consistent with previously reported data, ${ }^{6,22,36}$ a massive cell-cell fusion upon expression of all three variants of spike bearing the furin cleavage site was detected, and there was no syncytia formation in the samples transfected with $\Delta \mathrm{F}$ variants (Figure $2 \mathrm{C}$ ). This suggests that S-mediated syncytia formation inhibits lentiviral reporter expression in permissive cells; consequently, wild type $S$ protein cannot be used to assess cell-to-cell infection in the 293T/ACE2 cellular model. In summary, the possibility of measuring SARS-CoV-2 cell-to-cell infection using the intron-regulated luciferase vector was demonstrated, and the $\Delta \mathrm{F} \Delta \mathrm{C} 19$ mutant of $\mathrm{S}$ was selected for further study.

A

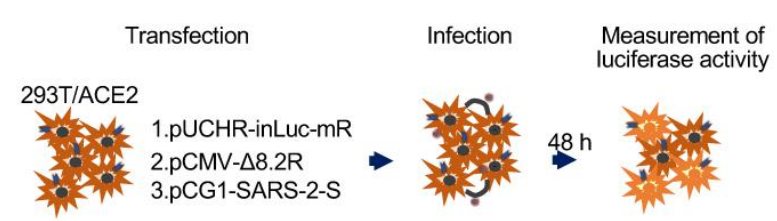

C
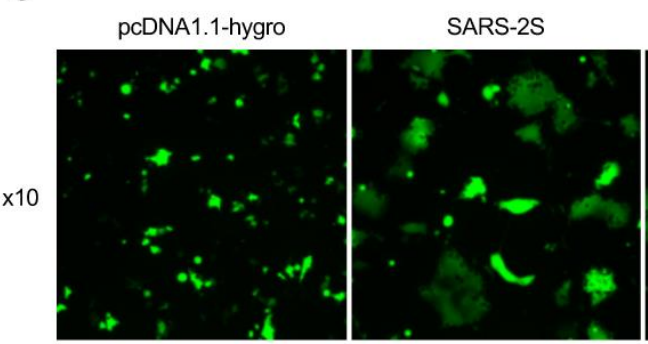

B
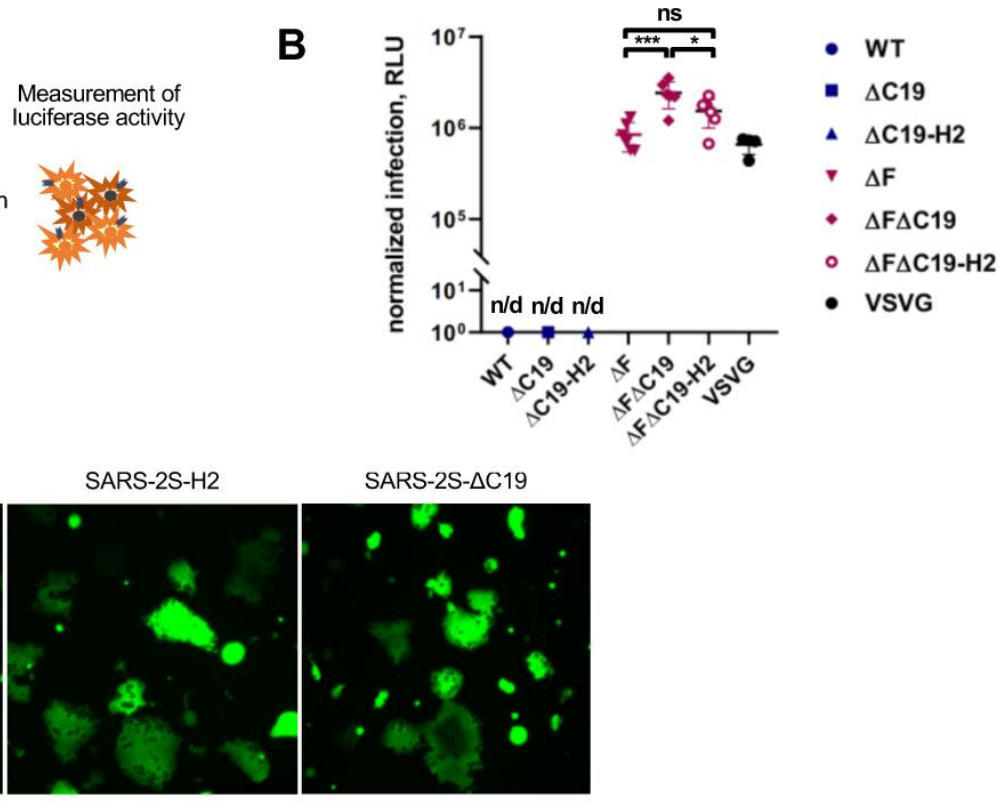

SARS-2S- $\Delta$ C19

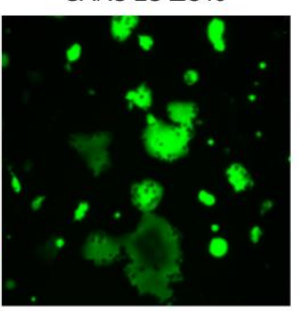

Figure 2. Establishing PV system for measurement of SARS-CoV-2 cell co-culture infection.

A. A schematic representation of SARS-CoV-2 one step transfection-infection assay in 293T/ACE2. B. The levels of infectivity measured using one step assay with different spike protein mutants. Samples with the intact furin cleavage site produced no signal above background ( $\mathrm{n} / \mathrm{d}$ ). The differences between $\Delta \mathrm{F}$ mutants were calculated by one-way ANOVA with the Tukey's multiple comparison test, and are significant at $p=0.0008(* * *)$ and $p=0.0457(*)$. C. Syncytia formation induced by wild-type or mutant SARS-CoV-2 S protein. 293 T cells were co-transfected 
with plasmids for expression of GFP and one of indicated variant of S protein with the intact furin cleavage site. At 24 hours posttransfection, cells were detached with 1 mM EDTA and mixed with 293T/ACE2 cells at 1:1 ratio for another 24 hours. Typical images of cells captured on epifluorescent microscope with a filters set for FITC are demonstrated.

\section{Comparative analysis of the neutralizing activity of convalescent sera in SARS-CoV-2 cell-free} and cell coculture pseudoviral infection tests.

Using the developed pseudoviral infection tests, side-by-side comparisons of the neutralizing activity of convalescent sera from COVID-19 patients in cell-free and cell coculture modes of infection were performed. To avoid possible biases that can be observed when a neutralizing agent is added at the time of infection initiation, neutralization tests were designed to allow either PVs or producer cells to be preincubated with a serum for 1 hour prior to the target cell addition (see schematic in Figure 3A). Specifically, cell-free PVs in the amount of $10 \mathrm{ng}$ of p24 were incubated with indicated serum dilutions in a total volume of $400 \mu \mathrm{l}$ of culture medium, and added to $8 \times 10^{4} 293$ T/ACE2 cells, seeded overnight in a 24-well plate. The levels of cell-free infection were estimated 48 hours later by measuring luciferase activity in cell lysates. In these experimental settings, the results with control samples were consistently reproduced at the level of $\sim 10^{6} \mathrm{RLU}$, giving an opportunity to detect a wide range of inhibitory activity. Five COVID-19 convalescent sera with high neutralizing activity were selected and evaluated in the cellfree infection test with $\triangle \mathrm{F} \Delta \mathrm{C} 19$. As shown in Figure $3 \mathrm{~A}$ and $\mathrm{E}$, all samples demonstrated $\mathrm{NT}_{50}$ in a range between $1 / 1500$ and 1/12000 dilution, whereas a non-immune serum had no inhibitory activity. Additionally, in order to determine whether the furin cleavage site mutation influenced neutralization titer, $\Delta C 19$ variant per se was tested. The inhibition rates against $\Delta C 19$ were slightly higher than those for $\Delta \mathrm{F} \Delta \mathrm{C} 19$ (Figure $3 \mathrm{~B}$ ), including $\mathrm{NT}_{50}$ values (Figure $3 \mathrm{C}$ ). Nevertheless, similar titration curves for all tested sera were observed with both $\Delta \mathrm{F} \Delta \mathrm{C} 19$ and $\Delta \mathrm{C} 19$. Thus, the $\Delta \mathrm{F}$ mutation did not dramatically change $\mathrm{S}$ protein neutralization in the cell-free test, but was absolutely necessary for measuring cell coculture infectivity and making the correct comparison between two types of infection.

The cell-to-cell neutralization test was designed to be as similar as possible to settings used for the cell-free PV inhibition analysis. To generate SARS-CoV-2 producer cells, non-permissive 293T cells were co-transfected with pCMV- $\Delta 8.2 \mathrm{R}$, pUCHR-inLuc-mR, and pCG1-SARS-2-S $\Delta \mathrm{F} \Delta \mathrm{C} 19$ plasmids, as described for cell-free infection. After 24 hours, cells were gently suspended using ethylenediaminetetraacetic acid (EDTA) and washed once with phosphate buffered saline (PBS); $2.6 \times 10^{4}$ transfected cells in $200 \mu$ culture medium were preincubated with a certain serum dilution for 1 hour at $+4^{\circ} \mathrm{C}$ and mixed with $5.6 \times 10^{4} 293$ T/ACE2 cells, giving a total of $8 \times 10^{4}$ cells in $0.4 \mathrm{ml}$ of culture medium. The cell mixture was placed in the wells of a 24-well plate and incubated for 48 hours before luciferase activity measurement (Figure 3D, schematic). The described format, and the resulting ratio of one producer cell to two target cells, provided the optimal sensitivity for measuring cell coculture infection and comparing it to cell-free infection in control samples. As shown in Figure 3D on the right, inhibition of SARS-CoV-2 cell coculture infection required high concentrations of convalescent sera, with $N T_{50}$ detected within the $1 / 100$ to $1 / 1100$ dilution range. Compared to the serum activities against cell-free infection, the neutralization capacities of the same sera against cell coculture infection were more than tenfold lower (Figure $3 \mathrm{E}$ ). Nonetheless, $\mathrm{NT}_{50}$ titers of individual serum samples measured in cell-free and cell-to-cell infection tests 
correlated to each other (Figure 3F), i.e., sera with a higher inhibitory titer detected in the cell-free infection test more effectively inhibited cell coculture infection.

In summary, by using the developed pseudoviral single cycle replication assay with the intronregulated reporter vector, it was demonstrated that SARS-CoV-2 cell coculture infection was much more resistant to neutralization by convalescent sera than infection with purified PVs.

A

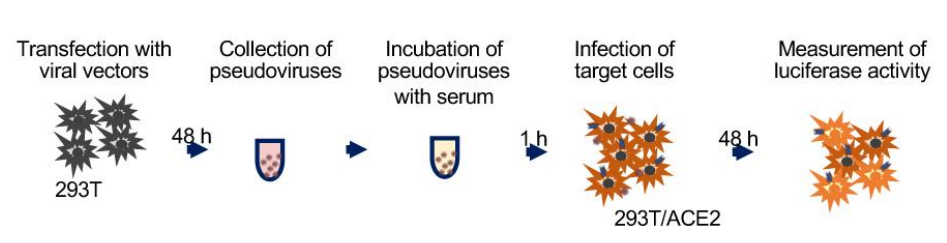

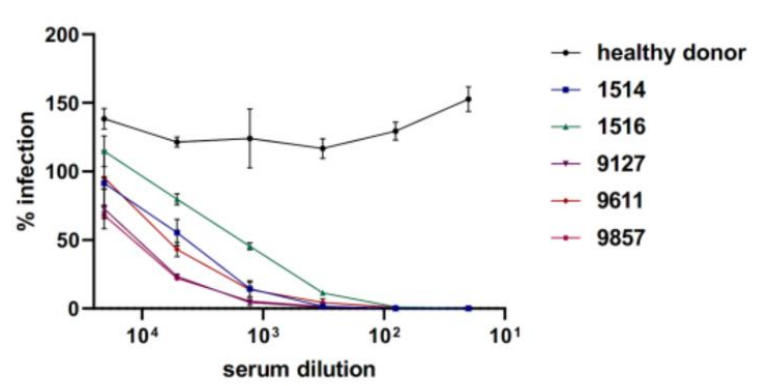

C
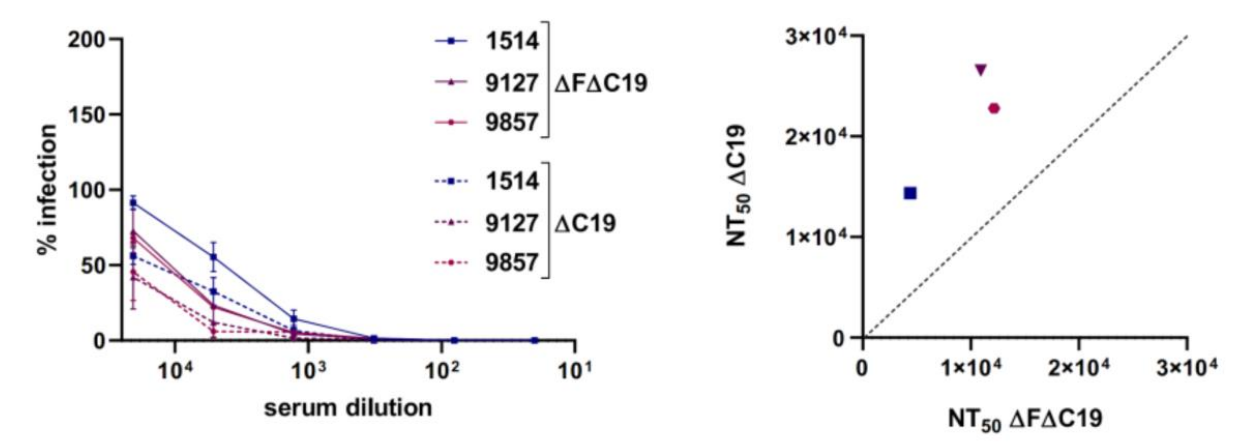

D
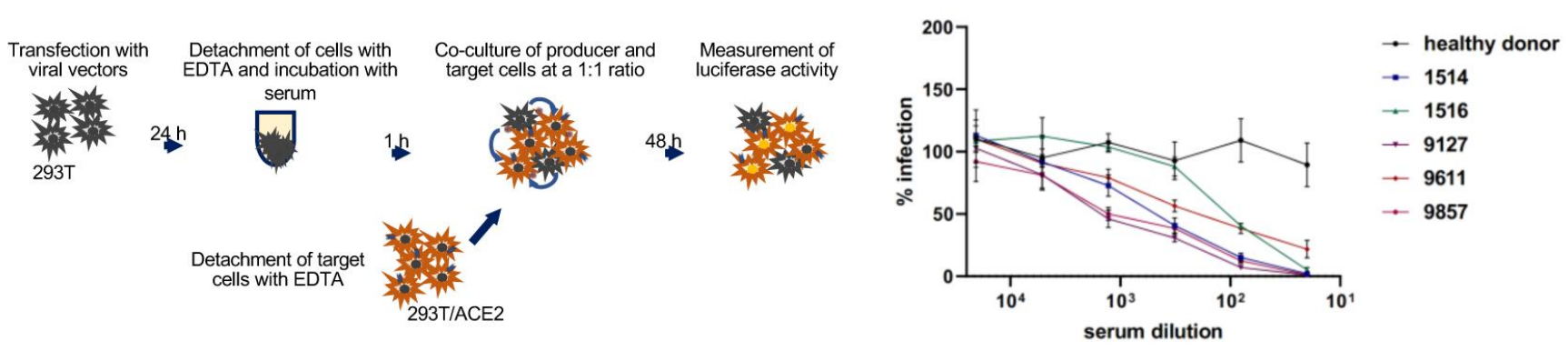

E

$\begin{array}{cccc}\text { serum } & \text { NT50 cell-free } & \text { NT50 cell-to-cell } & \begin{array}{c}\text { NT } 5_{50} \text { cell-to-cell / NT50 } \\ \text { cell-free }\end{array} \\ 1514 & 1 / 4385 & 1 / 462 & 9 \\ 1516 & 1 / 1494 & 1 / 100 & 15 \\ 9127 & 1 / 10928 & 1 / 1117 & 10 \\ 9611 & 1 / 5535 & 1 / 171 & 32 \\ 9857 & 1 / 12151 & 1 / 909 & 13\end{array}$

F

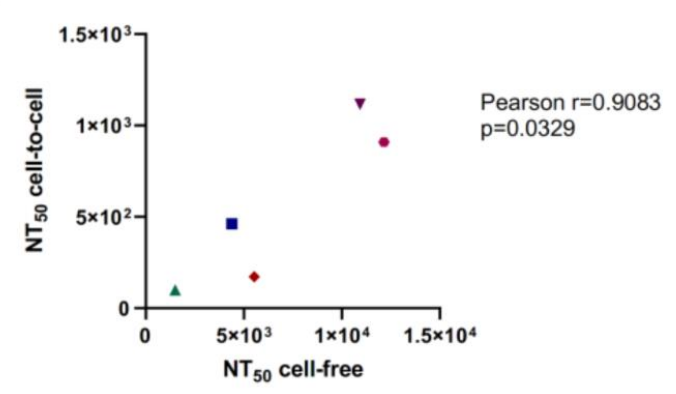

Figure 3. Neutralization activity of convalescent sera determined using SARS-CoV-2 PVs.

A. The experimental steps designed for cell-free neutrilization test. Viral particles pseudotyped with $\Delta \mathrm{F} \Delta \mathrm{C} 19$ were preincubated with human serum dillution for $1 \mathrm{~h}$ and added to the 293T/ACE2 target cells. The control RLU values obtained without serum were set at $100 \%$. The levels of infection detected in the presence of immune or non-immune serum were presented relative to control.. B. Neutralizing activity of convalescent sera against two indicated S protein mutants measured in a cell-free infection test. The assay was set up as in A. C. Correlation between $50 \%$ serum neutralizing titers ( $\mathrm{NT}_{50}$ ) obtained against $\mathrm{S} \triangle \mathrm{F} \Delta \mathrm{C} 19-\mathrm{PV}$ s and $\mathrm{S} \Delta \mathrm{C} 19-\mathrm{PV}$. D. A schematic illustrating cell coculture neutrilization assay setup (on the left) and neutrilization curves (on the right) obtained for indicated sera in this test. 293T cells transfected 
with viral vectors for 24 hours were detached with $1 \mathrm{mM}$ EDTA, incubated with a serially diluted serum for $1 \mathrm{~h}$, and co-cultured with 293 T/ACE2 cells at 1:1 ratio for $48 \mathrm{~h}$. Data were collected and presented as in $A$. The average results from three independent experiments \pm standard deviations are shown in A, B and D. E. Comparison of the 50\% serum neutralizing titers (NT ${ }_{50}$ ) obtained in cell-free and cell coculture infection tests with PVs. The values were extracted from data presented in A and D. F. The correlation between cell-free and cell coculture neutralizing titers detected for five convalescent sera.

\section{Neutralization potential of convalescent sera against replication-competent SARS-CoV-2}

Neutralization tests with PVs, although safe, have serious limitations, since they can only mimic the entry step of the viral life cycle. The mechanisms of viral assembly, egress, and transmission for the HIV and coronaviruses are very different, so the HIV-1 core proteins responsible for these processes - and used in this study's pseudoviral tests - cannot model SARS-CoV-2 cell-to-cell transmission. With an understanding of all the drawbacks of the developed tests, an investigation of whether the resistance of cell-to-cell transmission to antibody neutralization could be reproduced with a full replication-competent SARS-CoV-2 was conducted. To this end, Vero E6 monkey fibroblast cells were chosen for virus production, setting up cell-free and cell-to-cell infection. The 293T/ACE2 cells for this purpose were excluded, as they died quickly after infection with coronavirus, making viral stock generation or maintaining multiple cycles of replication impossible. To remain consistent with pseudoviral tests, the number of plated Vero cells were proportionally similar to what was used for 293T cells. Neutralization of cell-free infection was performed by preincubating $0.01 \mathrm{MOI}$ of SARS-CoV-2 strain hCoV19/Russia/Moscow_PMVL-4 with serially diluted convalescent sera for 1 hour, and then adding to the Vero cells, seeded in a 96-well plate overnight. At day five post infection, cytopathic effect (CPE) was measured using the MTT test. As shown in Figure 4A, all sera completely blocked SARS-CoV-2 replication at $1 / 100$ dilution; $N T_{50}$ values ranged from $1 / 400$ to $1 / 1400$. These values were lower than the corresponding $\mathrm{NT}_{50}$ determined in the pseudoviral test. This can be explained by the doses of PVs and virions used for the neutralization assays, which are difficult to compare or normalize. Nevertheless, the results of two cell-free assays correlated well to each other (Figure 4B).

Cell-to-cell SARS-CoV-2 spreading assay was initiated by infecting $2.6 \times 10^{4}$ Vero E6 cells with fully competent virus for 24 hours, followed by PBS washing and preincubating with a serum before it was added to $5.6 \times 10^{4}$ uninfected Vero E6 cells. The level of CPE was measured five days later using the MTT test. In stark contrast to the cell-free infectious test, the majority of the serum samples, even at minimal dilution, did not prevent cytopathic effect of fully-competent SARS-CoV-2 (Figure 4C), with the exception of serum 9611 , which at $1 / 20$ dilution displayed $\sim 50 \%$ inhibitory activity.

In conclusion, it was demonstrated that cell-to-cell spread of the fully competent wild type SARS263 CoV-2 is almost completely resistant to convalescent serum neutralization. This effect was even more 265 pronounced than the resistance detected using pseudoviruses.

A

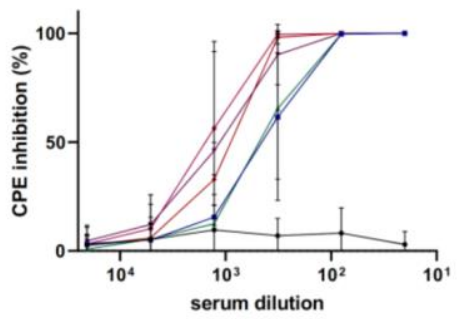

B

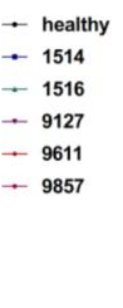

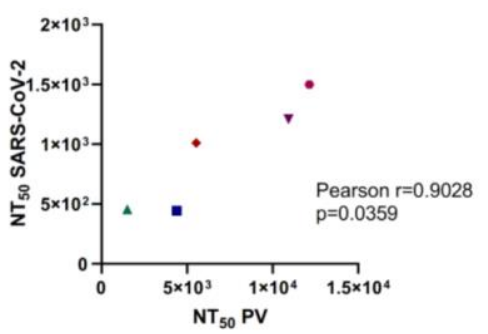

C

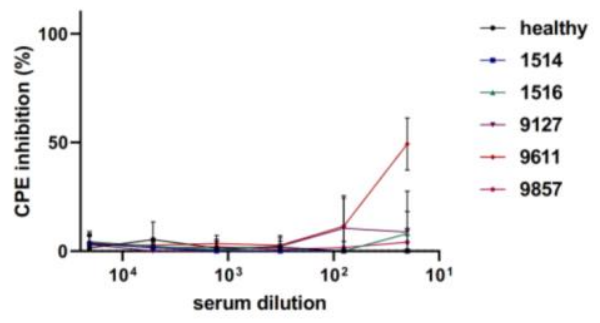


Figure 4. Neutralization activity of convalescent sera against replication-competent SARS-CoV-2.

A. Serum activity against the purified SARS-CoV-2 virions. Serial fourfold dilutions of serum samples from convalescent donors were incubated with SARS-CoV-2 and added to the VeroE6 cells. Cell survival was determined 5 days later by MTT test and expressed as a percentage of cytopathic effect (CPE) inhibition measured relative to untreated control, which was set at $100 \%$. B. Correlation between $\mathrm{NT}_{50}$ serum neutralizing titers measured with the $\triangle \mathrm{F} \Delta \mathrm{C} 19-\mathrm{PV}$ s and the live SARS-CoV-2. C. Serum neutrilizing activity against SARS-CoV-2 in cell coculture conditions. Serial fourfold dilutions of serum samples from convalescent donors were incubated with SARS-CoV-2 infected VeroE6 cells, which were then mixed with uninfected VeroE6 cells at 1:1 ratio. The results were collected and presented as in $A$. The average results from three independent experiments with standard deviations are shown in A and C.

\section{Discussion}

The fast and global spread of COVID-19 requires unprecedent efforts to control this pandemic. An important parameter of collective immunity, gained after SARS-CoV-2 infection or vaccination, is the neutralization activity of anti-spike antibodies. This reflects the degree to which the studied population is protected from the infection, and provides more adequate information than a titer of anti-spike antibodies measured by ELISA. However, this test requires strict BSL3 conditions in order to work with highly pathogenic full-length SARS-CoV-2, and it has not been widely utilized. In this respect, different PV systems are considered safe and acceptable for many labs, since they allow the completion of only one cycle of viral replication. The PVs have been adapted by many researchers to characterize the SARS-CoV-2 entry process, ${ }^{3,5,6,22,44,45}$ monitor the dynamics of neutralizing humoral immunity to SARS-CoV- $2,{ }^{14,46,47}$ and screen potential inhibitors. ${ }^{27,36}$

The aim of this study was to not just replicate a PV system but rather, develop a lentivirus-based PV test capable of measuring SARS-CoV-2 infection, both with purified PVs and in cell cocultures. The latter has not been appreciated previously or measured accurately. The use of identical vectors to initiate both types of infection makes comparative analysis of PV infectivity more rigorous. First, our system was optimized by pseudotyping PVs with S protein mutants. Since the cytoplasmic portion of the $S$ protein has an ER-Golgi retention signal needed for incorporation into coronaviruses that bud from endosomal membranes, ${ }^{48,49}$ but which may not be optimal for efficient pseudotyping of lenti- or retroviral particles that assemble predominantly at the plasma membrane, this signal should be removed. An early study on SARS-CoV by Giroglou et al. ${ }^{39}$ demonstrated that the C-terminally truncated spike increased PV infectivity, which led to the inclusion of this modification in the many subsequent PV systems developed for SARSCoV-2. ${ }^{6,32,41-43,50}$ The truncation of last $18-19,{ }^{43,50,51}$ or even 13 , amino acids ${ }^{41}$ enhanced PV infectivity from 10 to 100 fold. Consistent with the data reported above, our study has shown that the $\Delta$ C19 mutation improved cell-free infection by 15 fold, and one-step infection by 5 fold (Figure 1D and 2B). Nevertheless, a few studies did not find a substantial influence from $\Delta \mathrm{C} 19^{42}$ or point mutations in the ER retention signal ${ }^{33,52}$ on PV infectivity. In agreement with published papers, ${ }^{33,39,41}$ we did not observe substantial differences between wt and $\Delta \mathrm{C} 19 \mathrm{~S}$ protein levels expressed on the surface of PV producing cells (Figure 1G). Thus, the mechanism of enhanced infectivity for $\Delta \mathrm{C} 19 \mathrm{PVs}$ remains unclear, and can be related to improved incorporation of this mutant into PVs ${ }^{40,41,52}$ and/or stabilization of S1-S2 subunit interaction. ${ }^{41}$ Unlike simple truncation, the substitution of $\mathrm{C} 19$ with the most membrane-proximal cytoplasmic domain of gp41, $\triangle \mathrm{C} 19-\mathrm{H} 2^{40}$ did not alter PV infectivity in our tests. Crawford et al. substituted the cytoplasmic portion of the $S$ protein with the intracellular domain from influenza hemagglutinin, and reported no improvement in PV infectivity. ${ }^{33}$

Unlike SARS-CoV-1 S, the S protein of SARS-CoV-2 contains a furin cleavage site (F), located a little upstream of S1/S2 boundary. ${ }^{23}$ It became clear early on that the presence of $F$ increases Env-mediated cell-cell fusion, at least for in vitro experiments. ${ }^{22,53}$ However, the effects of $F$ on virus infectivity were contradictory, i.e., either a decrease ${ }^{44}$ or an increase ${ }^{45}$ in $\Delta F \mathrm{PV}$ infectivity relative to the wt $\mathrm{S}$ protein was reported. Finally, several groups found that infectivity depended on the cell target and, in particular, the 
314 entry site that the virus uses during infection. ${ }^{2,22,54}$ The latter is largely dependent on the $S$ protein 315 cleavage at S2' site by surface protease TMPRSS2 or lysosomal cathepsins. ${ }^{21}$ If cells express TMPRSS2 and 316 the virus enters via the plasma membrane, then $\triangle \mathrm{F}$ decreased $\mathrm{PV}$ infection ${ }^{22}$; whereas TMPRSS2-negative 317 target cells, such as the widely used 293T/ACE2 cells, were usually infected similarly ${ }^{2,22}$ or even better, 318 with $\triangle \mathrm{F} \mathrm{PVs},{ }^{44,45,52,54-56}$ depending on the mutations introduced at the $\mathrm{F}$ site. Consistent with these 319 reports, a 15-20 fold increase in infectivity with purified $\triangle F$ PVs in 293T/ACE2 cells was detected in this 320 study. Strikingly, one step transfection/infection using all variants of the $S$ protein with intact $F$ was 321 undetectable (Figure 2B), what was explained by massive syncytia formation induced by the $S$ protein 322 (Figure $2 \mathrm{C}$ ) and by blocking inLuc-mR transduction in fused cells. ${ }^{37}$ Thus, measurement of cell coculture

323 324 325 326 infection must adhere to the $\Delta \mathrm{F}$ variant of spike and be used in cell-free infection for comparative purposes. Summarizing this part of the study, the $\Delta \mathrm{F} \Delta \mathrm{C} 19$ mutant of the $\mathrm{S}$ protein was selected as the one providing the highest sensitivity to PV infection, both in cell-free and cell coculture experimental settings.

Next, the generated PV system was validated in a neutralization test with convalescent sera. As the $\Delta \mathrm{F}$ mutation is localized in the external part of the $\mathrm{S}$ protein, it can potentially influence serum neutralization activity. A comparison of the $\Delta \mathrm{F} \Delta \mathrm{C} 19$ and $\Delta \mathrm{C} 19$ mutants revealed that $\Delta \mathrm{F}$ required $\sim$ twofold higher serum concentration for PV neutralization than without $\Delta F$ (Figure $3 C$ ). This is consistent with the study by Johnson et al., ${ }^{57}$ and suggests that using the $\Delta \mathrm{F} \Delta \mathrm{C} 19$ mutant spike slightly underestimated the neutralization potential of sera, but not overestimated it. Using five selected COVID19 convalescent sera with high anti-spike titers, inhibitory activity was quantified against the $\Delta \mathrm{F} \Delta \mathrm{C} 19$ in cell-free and cell coculture modes of infection, and $\mathrm{NT}_{50}$ was calculated for all tested sera. It was demonstrated that all convalescent sera were at least tenfold less efficient in the neutralization of cell coculture infection, relative to inhibitory activity detected with purified PVs. Based on current literature, this is the first evidence that SARS-CoV-2 cell-to-cell infection is resistant to antibody neutralization. There is only one study in which infectivity of SARS-CoV-2 PVs was measured using a similar intron-containing reporter vector. It was based on Gaussia luciferase (Gluc) and produced minimal background activity. ${ }^{43}$ However, the authors of this study did not use it to measure cell coculture infection. Acknowledging that the validity of the results obtained in this study with lentiviral PVs could be heavily criticized, we conducted neutralization experiments on Vero E6 cells with live full-length SARS-CoV-2, at transmission settings that were as close as possible to those developed for the single round infection tests. The viral multiple replication assays not only confirmed the results with PVs, but also demonstrated the near complete resistance of SARS-CoV-2 cell coculture infection to neutralizing antibodies (Figure 4C). This phenomenon has been observed for a number of viruses, ${ }^{58-63}$ but has not been reported for coronaviruses.

Some respiratory viruses have been shown to utilize cell-to-cell transmission. Examples include induction of intercellular extensions by the influenza virus, PIV5, ${ }^{64,65} \mathrm{HMPV},{ }^{58,66}$ and $\mathrm{RSV}^{67}$, usage of intercellular membrane pores by the measles virus, which is also able to infect airway epithelium. ${ }^{68}$ Coronaviruses extensively reorganize not only the ER-Golgi network but also change plasma membrane characteristics, inducing formation ruffles and filopodia. ${ }^{69}$ Ogando et al. observed that Vero E6 cells infected with SARS-CoV-2 alter their morphology by forming long filopodia with budding viruses. ${ }^{70}$ The $^{2}$ study on Caco- 2 cells by Bouhaddou et al. showed that the N protein of SARS-CoV-2 interacts with the kinase CK2 that leads to cytoskeleton reorganization and filopodia formation. ${ }^{71}$ Using scanning electron microscopy, Caldas et al. visualized thin protrusions between Vero cells with adherent virions. ${ }^{72}$ Whether the observed intercellular contacts play a role in SARS-CoV-2 cell-to-cell transmission in vivo is not known. 
357 Massive cell-cell fusion induced by SARS-CoV-2 has been observed in cell culture ${ }^{22,53}$ and in human 358 organoids, ${ }^{73,74}$ as well as in post-mortem material. ${ }^{75-79}$ However, the biological significance of syncytia 359 formation in vivo remains uncertain. The most logical mechanism that would protect SARS-CoV-2 cell-to360 cell transmission from antibody neutralization is a tight cellular contact with a synaptic cleft where the 361 virus buds, and where large immunoglobulins may have difficulty penetrating. This mechanism has been 362 implied for retroviral infectious synapses. ${ }^{80}$ However, such structures have not been described for SARSCoV-2, which transmits in airway epithelium.

In conclusion, we developed lentivirus-based single round pseudoviral infection assays suitable for quantitatively measuring SARS-CoV-2 entry in cell-free and cell coculture conditions. Using this system, as well as the SARS-CoV-2 spreading assay, our study has shown that cell-to-cell infection of SARS-CoV-2 is considerably more resistant to serum neutralization than infection with purified viral particles. These results underline the importance of SARS-CoV-2 cell-to-cell transmission for virus biology, immune protection, and the development of entry inhibitors. Further experiments are required to understand the mechanisms of this resistance. The developed assays are safe, easily reproducible, and believed to be more appropriate for validating the neutralization activity of antibodies, peptides, and small molecules than the PV tests described earlier.

\section{Materials and methods}

Cell lines. The human embryonic kidney 293T cells were obtained through NIH AIDS Research and Reference Reagent Program. Vero E6 cells were obtained from ATCC (CRL-1586). All cell lines were cultured in high glucose Dulbecco's modified Eagle's medium (DMEM) (Sigma-Aldrich, USA) with sodium pyruvate, sodium bicarbonate, $10 \%$ fetal calf serum (FCS), $2 \mathrm{mM}$ glutamine and $40 \mu \mathrm{g} / \mathrm{ml}$ gentamicin at $37^{\circ} \mathrm{C}$ and $5 \% \mathrm{CO} 2$. The cells have been tested negative for mycoplasma contamination.

Human serum samples. All serum samples were derived from the human serum biobank of the Gamaleya Center for Epidemiology and Microbiology. Study was approved by the Local ethic committee of the Moscow First Infectious Disease Hospital (Protocol \#2 dated 2021-01-22).

Plasmid construction. The plasmid pCG1-SARS-2-S coding for the codon-optimized S-protein was kindly provided by Prof. Dr. Stefan Pöhlmann (Infection biology unit of the German Primate Center, Leibniz Institute for Primate Research). C-terminal truncation of the S-protein ( $\Delta C 19)$, addition of 8 amino acids from the HIV gp41 $(\mathrm{H} 2)$ and mutation of the furin cleavage site PRRA $\rightarrow A(\triangle A)$ were introduced by PCR with Pfu polymerase (Sibenzyme, Russia) and verified by sequencing. The HIV-1 (strain NL4-3) packaging plasmids pCMV-dR8-2 (\# 12263), vector pCMV-VSV-G for expression of the protein G from vesicular stomatitis virus (\# 8454) were obtained from Addgene; reporter plasmids pUCHR-inLuc-mR and pUCHR-IR-GFP were described previously ${ }^{37,38}$. The plasmid pUCHR-hACE2 was generated by subcloning the ACE2 coding sequence from the pCG1-hACE2 plasmid obtained from Prof. Dr. Stefan Pöhlmann (Infection biology unit of the German Primate Center, Leibniz Institute for Primate Research) into lentiviral vector pUCHR.

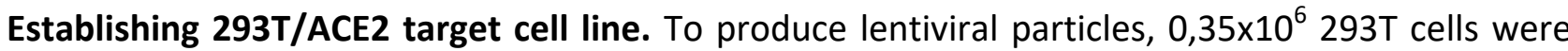
plated in 1 well of a 6 -well plate in $2.5 \mathrm{ml}$ of growth medium. The next day, the cells were transfected with $0.66 \mu \mathrm{g}$ of pCMV-dR8-2, $0.88 \mu \mathrm{g}$ of pUCHR-hACE2, and $0.22 \mu \mathrm{g}$ pCMV-VSVG using Lipofectamine 2000 (ThermoFisher Scientific) according to the manufacturer's instruction. At $48 \mathrm{~h}$ posttransfection, supernatants with pseudoviruses were cleared through $0.45 \mu \mathrm{m}$ pore size filters and used for transduction. $8 \times 10^{4} 293 \mathrm{~T}$ cells per well were plated in a 24 -well plate in $500 \mu$ l of growth medium. In $24 \mathrm{~h}$, 
400

401

402

403

404

405

406

407

408

409

410

411

412

413

414

415

416

417

418

419

420

421

422

423

424

425

426

427

428

429

430

431

432

433

434

435

436

437

438

439

440

441

442

serially diluted lentiviral particles were added to the cells for. The percentage of ACE2-positive cells was analyzed by flow cytometry at $48 \mathrm{~h}$ postinfection. The sample with approximately $30 \%$ level of transduction was selected for further isolation using Sony MA900 (Sony Biotechnology, San Jose, CA, USA) cell sorter. The cells were expanded and sorted once again to enrich ACE2 positive population more than 98\%.

Generation of SARS-CoV-2 pseudotyped viral particles. $2,5 \times 10^{6} 293$ T cells were plated in a $10-\mathrm{cm}$ dish in $10 \mathrm{ml}$ of growth medium. The next day, the cells were transfected with $5 \mu \mathrm{g}$ pCMV-dR8-2, 6.67 $\mu \mathrm{g}$ pUCHR-inLuc-mR or pUCHR-IR-GFP, and $3.33 \mu \mathrm{g}$ of the S-protein coding plasmid using Lipofectamine 2000 (ThermoFisher Scientific) according to the manufacturer's instruction. $48 \mathrm{~h}$ posttransfection, pseudoviruses were cleared through $0.45 \mu \mathrm{m}$ filters, concentrated by centrifugation at $20000 \mathrm{~g}, 4^{\circ} \mathrm{C}, 2.5 \mathrm{~h}$, aliquoted and stored at $-80^{\circ} \mathrm{C}$. Pseudoviruses were titrated on 293 T/ACE2 cells and assessed by flow cytometry (GFP) or by luciferase assay (inLuc). p24 level for each preparation was measured by the HIV p24 ELISA kit (Vector-Best, Russia).

One step transfection/infection assay. A single-round transfection/infection test was performed in a 24 well format. $8 \times 10^{4} 293$ T/ACE2 cells per well plated in $500 \mu \mathrm{l}$ of growth medium $24 \mathrm{~h}$ in advance were transfected with $0.217 \mu \mathrm{g}$ pCMV-dR8-2, $0.288 \mu \mathrm{g}$ pUCHR-inLuc-mR, and 0.144 $\mu \mathrm{g}$ pCG1-SARS-2-S or its derivative or $0.072 \mu \mathrm{g}$ pCMV-VSVG as a control using Lipofectamine 2000 (ThermoFisher Scientific) according to the manufacturer's instruction. $48 \mathrm{~h}$ posttransfection, culture supernatants were removed, centrifuged and used for p24 calculation by ELISA. The cells were lysed with the GLO lysis buffer (\# E2661, Promega), luciferase activity was determined by the Bright-Glo ${ }^{\mathrm{TM}}$ Luciferase Assay System (\# E2620, Promega) using GloMax ${ }^{\circledR}$ 20/20 Luminometer (Promega).

Detection of syncytia formation. $8 \times 10^{4} 293$ T cells per well were plated in a 24-well plate in $500 \mu \mathrm{l}$ of growth medium. After $24 \mathrm{~h}$, the cells were transfected with 0,5 $\mu \mathrm{g}$ pCMV-GFPt and 0,3 $\mu \mathrm{g}$ pCG1-SARS2-S, pCG1-SARS-2-SdC19 or pCG1-SARS-2-SdC19-H2 using Lipofectamine 2000 (ThermoFisher Scientific) according to the manufacturer's instruction. The next day, the transfected cells and 293T/ACE2 cells were detached with $1 \mathrm{mM}$ EDTA, mixed at the ratio of 1:1 and plated in wells of a 24-well plate with the total number of $10^{5}$ cells per well. Images of live cells were acquired by the Nikon eclipse Ti microscope at the x10 magnification $24 \mathrm{~h}$ later.

Single-cycle cell-free infection. $8 \times 10^{4} 293$ T/ACE2 cells per well were plated in a 24-well plate in $400 \mu \mathrm{l}$ of growth medium. The following day, the whole volume was replaced with $400 \mu \mathrm{l}$ of medium containing pseudoviruses. Infection level was determined $48 \mathrm{~h}$ later by luciferase assay or flow cytometry. To measure neutralizing activity of sera from COVID-19 patients, sera were serially four-fold diluted in growth medium and preincubated with pseudoviruses in the total volume of $400 \mu \mathrm{l}$ for $1 \mathrm{~h}$ at room temperature before addition to target cells.

Single-cycle cell coculture infection. To generate pseudovirus-producing cells, $9 \times 10^{5} 293$ cells were plated in a $6-\mathrm{cm}$ dish in $5 \mathrm{ml}$ of growth medium. The next day, the cells were transfected with 1.67 $\mu \mathrm{g}$ pCMV-dR8-2, $2.22 \mu \mathrm{g}$ pUCHR-inLuc-mR, and $1.11 \mu \mathrm{g}$ pCG1-SARS-2-SdFdC19 using Lipofectamine 2000 (ThermoFisher Scientific) according to the manufacturer's instruction. $24 \mathrm{~h}$ posttransfection, producer cells were detached with $1 \mathrm{mM}$ EDTA and washed twice with PBS. $2.6 \times 10^{4}$ cells were mixed with serial four-fold dilutions of sera in the total volume of $200 \mu \mathrm{l}$ and incubated for $1 \mathrm{~h}$ at $4^{\circ} \mathrm{C}$. Next, they were mixed with $5.4 \times 10^{4}$ target 293T/ACE2 cells detached with $1 \mathrm{mM}$ EDTA and resuspended in $200 \mu \mathrm{l}$ of medium. Cell mixture was plated in a 24 -well plate and co-cultured in the $400 \mu$ l volume of medium. Luciferase activity was determined $48 \mathrm{~h}$ later. 
SARS-CoV-2 virus stock. SARS-CoV-2 strain hCoV-19/Russia/Moscow_PMVL-4 (EPI_ISL_470898) ${ }^{81}$, was amplified and titrated on Vero E6 cells. Viral titers were determined as $\mathrm{TCID}_{50}$ by endpoint dilution assay. All experiments with live SARS-CoV-2 were performed in biosafety level 3 facility (BSL-3).

Cell-free SARS-CoV-2 spreading assay. Vero E6 cells were plated at $8 \times 10^{4}$ cells/well into 96 -well plates the day prior to experiments. Serum samples were serially four-fold diluted in growth medium, mixed with MOI 0.01 of SARS-CoV-2 and incubated for $1 \mathrm{~h}$ at $37^{\circ} \mathrm{C}$. The mixture was then added to Vero E6 cells and incubated for 5 days at $37^{\circ} \mathrm{C}$. Cytopathic effect (CPE) was determined by MTT assay ${ }^{82,83}$.

Cell-to-cell SARS-CoV-2 spreading assay. Vero E6 cells were plated into T25 cell culture flask and infected with SARS-CoV-2 at MOI 0.01. The next day, infected cells were detached with Trypsin/EDTA solution (Gibco, USA), washed twice with PBS, mixed with serial four-fold dilutions of sera at $2.6 \times 10^{4}$ cells per sample and incubated for $1 \mathrm{~h}$ at $37^{\circ} \mathrm{C}$. The mixture was then combined with $5.4 \times 10^{4}$ uninfected Vero E6 cells in 96-well plates in the total volume of $200 \mu \mathrm{l}$ and incubated for 5 days at $37^{\circ} \mathrm{C}$. CPE was determined by MTT assay.

Flow cytometry. To measure S protein expression on the surface of 293T pseudovirus producing cells, $3 \times 10^{5}$ live cells were incubated with the serum from a convalescent donor at the 1:100 dilution in PSB for 30 min followed by the incubation with secondary anti-human IgG antibodies conjugated with PE (1:250, \# H10104, ThermoFisher Scientific) for 30 min. ACE2 expression was assessed by staining cells with polyclonal rabbit antibodies against human ACE2 (PAB886Hu01, Cloud-Clone Corp.) followed by secondary anti-rabbit antibodies conjugated with PE (1:250, \# P-2771MP, ThermoFisher Scientific). Samples were analyzed on CytoFLEX S flow cytometer (Beckman Coulter). FlowJo LLC software was used for histogram visualization.

Data analysis. The data were analyzed and visualized using GraphPad Prism 8 Software. NT 50 values were calculated using nonlinear regression curve fit to normalized data expressed as a percentage of infectivity inhibition.

\section{Acknowledgements}

This work was supported by the grant 075-15-2019-1661 from the Ministry of Science and Higher Education of the Russian Federation.

The authors declare no financial or non-financial interests.

\section{Competing interests}

\section{References}

1. Zhou, P. et al. A pneumonia outbreak associated with a new coronavirus of probable bat origin. Nature 579, 270-273 (2020).

2. Walls, A. C. et al. Structure, Function, and Antigenicity of the SARS-CoV-2 Spike Glycoprotein. Cell 181, 281-292.e6 (2020).

3. Letko, M., Marzi, A. \& Munster, V. Functional assessment of cell entry and receptor usage for SARS-CoV-2 and other lineage B betacoronaviruses. Nat. Microbiol. 5, 562-569 (2020).

4. Zhu, N. et al. Morphogenesis and cytopathic effect of SARS-CoV-2 infection in human airway epithelial cells. Nat. Commun. 11, 1-8 (2020).

5. Hoffmann, M. et al. SARS-CoV-2 Cell Entry Depends on ACE2 and TMPRSS2 and Is Blocked by a Clinically Proven Protease Inhibitor. Cell 181, 271-280.e8 (2020).

6. Ou, X. et al. Characterization of spike glycoprotein of SARS-CoV-2 on virus entry and its immune cross-reactivity with SARS-CoV. Nat. Commun. 11, 1-12 (2020).

7. Trypsteen, W., Van Cleemput, J., van Snippenberg, W., Gerlo, S. \& Vandekerckhove, L. On the whereabouts of SARS-CoV-2 in the human body: A systematic review. PLoS Pathog. 16, e1009037 (2020).

8. Müller, J. A. et al. SARS-CoV-2 infects and replicates in cells of the human endocrine and exocrine pancreas. Nat. Metab. 3, 149-165 (2021).

9. Daly, J. L. et al. Neuropilin-1 is a host factor for SARS-CoV-2 infection. Science (80-. ). 370, 861-865 (2020).

10. Cantuti-Castelvetri, L. et al. Neuropilin-1 facilitates SARS-CoV-2 cell entry and infectivity. Science (80-. ). 370, (2020).

11. Wang, S. et al. AXL is a candidate receptor for SARS-CoV-2 that promotes infection of pulmonary and bronchial epithelial cells. Cell Res. 31, 126-140 (2021). 
Wang, K. et al. CD147-spike protein is a novel route for SARS-CoV-2 infection to host cells. Signal Transduct. Target. Ther. 5, 1-10 (2020).

13. Shilts, J., Crozier, T. W. M., Greenwood, E. J. D., Lehner, P. J. \& Wright, G. J. No evidence for basigin/CD147 as a direct SARS-CoV-2 spike binding receptor. Sci. Rep. 11, 413 (2021).

14. Hoffmann, M. et al. SARS-CoV-2 variants B.1.351 and P.1 escape from neutralizing antibodies. Cell (2021). doi:10.1016/j.cell.2021.03.036

15. Rey, F. A. \& Lok, S. M. Common Features of Enveloped Viruses and Implications for Immunogen Design for Next-Generation Vaccines. Cell 172, 1319-1334 (2018).

16. Cohen, F. S. \& Melikyan, G. B. The energetics of membrane fusion from binding, through hemifusion, pore formation, and pore enlargement. Journal of Membrane Biology 199, 1-14 (2004).

17. Li, F. Structure, Function, and Evolution of Coronavirus Spike Proteins. Annu. Rev. Virol. 3, 237-261 (2016).

18. Wrapp, D. et al. Cryo-EM structure of the 2019-nCoV spike in the prefusion conformation. Science (80-. ). 367, (2020).

19. Yao, H. et al. Molecular Architecture of the SARS-CoV-2 Virus. Cell 183, 730-738.e13 (2020).

20. Benton, D. J. et al. Receptor binding and priming of the spike protein of SARS-CoV-2 for membrane fusion. Nature 588, 327-330 (2020).

21. Millet, J. K. \& Whittaker, G. R. Host cell proteases: Critical determinants of coronavirus tropism and pathogenesis. Virus Res. 202, 120-134 (2015).

22. Hoffmann, M., Kleine-Weber, H. \& Pöhlmann, S. A Multibasic Cleavage Site in the Spike Protein of SARS-CoV-2 Is Essential for Infection of Human Lung Cells. Mol. Cell 78, 779-784.e5 (2020).

23. Andersen, K. G., Rambaut, A., Lipkin, W. I., Holmes, E. C. \& Garry, R. F. The proximal origin of SARS-CoV-2. Nat. Med. 26, 450-452 (2020).

24. Wu, F. et al. Neutralizing antibody responses to SARS-CoV-2 in a COVID-19 recovered patient cohort and their implications. medRxiv 2020.03.30.20047365 (2020). doi:10.1101/2020.03.30.20047365

25. Chen, X. et al. Human monoclonal antibodies block the binding of SARS-CoV-2 spike protein to angiotensin converting enzyme 2 receptor. Cellular and Molecular Immunology 17, 647-649 (2020).

26. Tai, W. et al. Characterization of the receptor-binding domain (RBD) of 2019 novel coronavirus: implication for development of RBD protein as a viral attachment inhibitor and vaccine. Cell. Mol. Immunol. 17, 613-620 (2020)

27. Lei, C. et al. Neutralization of SARS-CoV-2 spike pseudotyped virus by recombinant ACE2-Ig. Nat. Commun. 11, 2070 (2020).

28. Xia, S. et al. Inhibition of SARS-CoV-2 (previously 2019-nCoV) infection by a highly potent pan-coronavirus fusion inhibitor targeting its spike protein that harbors a high capacity to mediate membrane fusion. Cell Res. 30, 343-355 (2020).

29. Pinto, D. et al. Cross-neutralization of SARS-CoV-2 by a human monoclonal SARS-CoV antibody. Nature 583, $290-295$ (2020).

30. Quinlan, B. D. et al. The SARS-CoV-2 receptor-binding domain elicits a potent neutralizing response without antibody-dependent enhancement. bioRxiv 2020.04.10.036418 (2020). doi:10.1101/2020.04.10.036418

31. Nie, J. et al. Establishment and validation of a pseudovirus neutralization assay for SARS-CoV-2. Emerg. Microbes Infect. 9, 680-686 (2020).

32. Xiong, H. L. et al. Robust neutralization assay based on SARS-CoV-2 S-protein-bearing vesicular stomatitis virus (VSV) pseudovirus and ACE2-overexpressing BHK21 cells. Emerg. Microbes Infect. 9, 2105-2113 (2020).

33. Crawford, K. H. D. et al. Protocol and Reagents for Pseudotyping Lentiviral Particles with SARS-CoV-2 Spike Protein for Neutralization Assays. Viruses 12, 513 (2020).

34. Nie, J. et al. Quantification of SARS-CoV-2 neutralizing antibody by a pseudotyped virus-based assay. Nat. Protoc. 15, 3699-3715 (2020).

35. Riepler, L. et al. Comparison of Four SARS-CoV-2 Neutralization Assays. Vaccines 9, 13 (2020).

36. Zhu, Y., Yu, D., Yan, H., Chong, H. \& He, Y. Design of Potent Membrane Fusion Inhibitors against SARS-CoV-2, an Emerging Coronavirus with High Fusogenic Activity. J. Virol. 94, (2020).

37. Mazurov, D., Ilinskaya, A., Heidecker, G., Lloyd, P. \& Derse, D. Quantitative Comparison of HTLV-1 and HIV-1 Cell-to-Cell Infection with New Replication Dependent Vectors. PLoS Pathog. 6, e1000788 (2010).

38. Shunaeva, A. et al. Improvement of HIV-1 and Human T Cell Lymphotropic Virus Type 1 Replication-Dependent Vectors via Optimization of Reporter Gene Reconstitution and Modification with Intronic Short Hairpin RNA. J. Virol. 89, 10591-10601 (2015).

39. Giroglou, T. et al. Retroviral Vectors Pseudotyped with Severe Acute Respiratory Syndrome Coronavirus S Protein. J. Virol. 78, 90079015 (2004).

40. Moore, M. J. et al. Retroviruses Pseudotyped with the Severe Acute Respiratory Syndrome Coronavirus Spike Protein Efficiently Infect Cells Expressing Angiotensin-Converting Enzyme 2. J. Virol. 78, 10628-10635 (2004).

41. Yu, J. et al. Deletion of the SARS-CoV-2 Spike Cytoplasmic Tail Increases Infectivity in Pseudovirus Neutralization Assays. J. Virol. (2021). doi:10.1128/JVI.00044-21

42. Neerukonda, S. N. et al. Establishment of a well-characterized SARS-CoV-2 lentiviral pseudovirus neutralization assay using $293 \mathrm{~T}$ cells with stable expression of ACE2 and TMPRSS2. PLoS One 16, e0248348 (2021).

43. Johnson, M. C. et al. Optimized Pseudotyping Conditions for the SARS-COV-2 Spike Glycoprotein. J. Virol. 94, (2020).

44. Shang, J. et al. Cell entry mechanisms of SARS-CoV-2. Proc. Natl. Acad. Sci. 202003138 (2020). doi:10.1073/pnas.2003138117

45. Zhang, L. et al. SARS-CoV-2 spike-protein D614G mutation increases virion spike density and infectivity. Nat. Commun. 11, 1-9 (2020).

46. Garcia-Beltran, W. F. et al. Multiple SARS-CoV-2 variants escape neutralization by vaccine-induced humoral immunity. Cell (2021). doi:10.1016/j.cell.2021.03.013

47. Dan, J. M. et al. Immunological memory to SARS-CoV-2 assessed for up to 8 months after infection. Science (80-. ). 371, (2021). 
McBride, C. E., Li, J. \& Machamer, C. E. The Cytoplasmic Tail of the Severe Acute Respiratory Syndrome Coronavirus Spike Protein Contains a Novel Endoplasmic Reticulum Retrieval Signal That Binds COPI and Promotes Interaction with Membrane Protein. J. Virol. 81, 2418-2428 (2007).

49. V'kovski, P., Kratzel, A., Steiner, S., Stalder, H. \& Thiel, V. Coronavirus biology and replication: implications for SARS-CoV-2. Nature Reviews Microbiology 19, 155-170 (2020).

50. Schmidt, F. et al. Measuring SARS-CoV-2 neutralizing antibody activity using pseudotyped and chimeric viruses. J. Exp. Med. 217, (2020).

51. Fu, X., Tao, L. \& Zhang, X. Comprehensive and systemic optimization for improving the yield of SARS-CoV-2 spike pseudotyped virus. Mol. Ther. - Methods Clin. Dev. 20, 350-356 (2021).

52. Nguyen, H. T. et al. Spike Glycoprotein and Host Cell Determinants of SARS-CoV-2 Entry and Cytopathic Effects. J. Virol. 95, 23042324 (2020).

53. Xia, S. et al. The role of furin cleavage site in SARS-CoV-2 spike protein-mediated membrane fusion in the presence or absence of trypsin. Signal Transduction and Targeted Therapy 5, 1-3 (2020).

54. Hansen, J. et al. Studies in humanized mice and convalescent humans yield a SARS-CoV-2 antibody cocktail. Science (80-. ). 369, 1010-1014 (2020).

55. Peacock, T. P. et al. The furin cleavage site of SARS-CoV-2 spike protein is a key determinant for transmission due to enhanced replication in airway cells. bioRxiv 2020.09.30.318311 (2020). doi:10.1101/2020.09.30.318311

56. Zhu, Y. et al. The S1/S2 boundary of SARS-CoV-2 spike protein modulates cell entry pathways and transmission. bioRxiv 2020.08.25.266775 (2020). doi:10.1101/2020.08.25.266775

57. Johnson, B. A. et al. Loss of furin cleavage site attenuates SARS-CoV-2 pathogenesis. Nature 591, 293-299 (2021).

58. El Najjar, F. et al. Human metapneumovirus Induces Reorganization of the Actin Cytoskeleton for Direct Cell-to-Cell Spread. PLoS Pathog. 12, (2016)

59. Brimacombe, C. L. et al. Neutralizing Antibody-Resistant Hepatitis C Virus Cell-to-Cell Transmission. J. Virol. 85, 596-605 (2011).

60. Martin, N. et al. Virological Synapse-Mediated Spread of Human Immunodeficiency Virus Type 1 between T Cells Is Sensitive to Entry Inhibition. J. Virol. 84, 3516-3527 (2010).

61. Merwaiss, F., Czibener, C. \& Alvarez, D. E. Cell-to-Cell Transmission Is the Main Mechanism Supporting Bovine Viral Diarrhea Virus Spread in Cell Culture. J. Virol. 93, (2018).

62. Xiao, F. et al. Hepatitis C Virus Cell-Cell Transmission and Resistance to Direct-Acting Antiviral Agents. PLoS Pathog. 10, e1004128 (2014).

63. Mothes, W., Sherer, N. M., Jin, J. \& Zhong, P. Virus Cell-to-Cell Transmission. J. Virol. 84, 8360-8368 (2010).

64. Roberts, K. L., Manicassamy, B. \& Lamb, R. A. Influenza A Virus Uses Intercellular Connections To Spread to Neighboring Cells. J. Virol. 89, 1537-1549 (2015).

65. Kumar, A. et al. Influenza virus exploits tunneling nanotubes for cell-to-cell spread. Sci. Rep. 7, (2017).

66. Kinder, J. T. et al. Respiratory Syncytial Virus and Human Metapneumovirus Infections in Three-Dimensional Human Airway Tissues Expose an Interesting Dichotomy in Viral Replication, Spread, and Inhibition by Neutralizing Antibodies. J. Virol. 94, (2020).

67. Mehedi, M. et al. Actin-Related Protein 2 (ARP2) and Virus-Induced Filopodia Facilitate Human Respiratory Syncytial Virus Spread. PLoS Pathog. 12, (2016).

68. Singh, B. K. et al. The Nectin-4/Afadin Protein Complex and Intercellular Membrane Pores Contribute to Rapid Spread of Measles Virus in Primary Human Airway Epithelia. J. Virol. 89, 7089-7096 (2015).

69. Ng, M. L. et al. Topographic changes in SARS coronavirus-infected cells during late stages of infection. Emerg. Infect. Dis. 10, 19071914 (2004).

70. Ogando, N. S. et al. SARS-coronavirus-2 replication in Vero E6 cells: Replication kinetics, rapid adaptation and cytopathology. J. Gen. Virol. 101, 925-940 (2020).

71. Bouhaddou, M. et al. The Global Phosphorylation Landscape of SARS-CoV-2 Infection. Cell (2020). doi:10.1016/j.cell.2020.06.034

72. Caldas, L. A. et al. Ultrastructural analysis of SARS-CoV-2 interactions with the host cell via high resolution scanning electron microscopy. Sci. Rep. 10, 16099 (2020).

73. Zang, R. et al. TMPRSS2 and TMPRSS4 promote SARS-CoV-2 infection of human small intestinal enterocytes. Sci. Immunol. 5, (2020).

74. Mykytyn, A. Z. et al. Sars-cov-2 entry into human airway organoids is serine protease-mediated and facilitated by the multibasic cleavage site. Elife 10, 1-23 (2021).

75. Xu, Z. et al. Pathological findings of COVID-19 associated with acute respiratory distress syndrome. Lancet Respir. Med. 8, 420-422 (2020).

76. Bussani, R. et al. Persistence of viral RNA, pneumocyte syncytia and thrombosis are hallmarks of advanced COVID-19 pathology. EBioMedicine 61, (2020).

77. Sanders, D. W. et al. SARS-CoV-2 requires cholesterol for viral entry and pathological syncytia formation. bioRxiv 2020.12.14.422737 (2020). doi:10.1101/2020.12.14.422737

78. Bryce, C. et al. Pathophysiology of SARS-CoV-2: Targeting of endothelial cells renders a complex disease with thrombotic microangiopathy and aberrant immune response. The Mount Sinai COVID-19 autopsy experience. medRxiv 2020.05.18.20099960 (2020). doi:10.1101/2020.05.18.20099960

79. Rockx, B. et al. Comparative pathogenesis of COVID-19, MERS, and SARS in a nonhuman primate model. Science (80-. ). 368, 10121015 (2020).

80. Dufloo, J., Bruel, T. \& Schwartz, O. HIV-1 cell-to-cell transmission and broadly neutralizing antibodies. Retrovirology 15, 51 (2018). 81. Antipova, N. V. et al. Establishment of Murine Hybridoma Cells Producing Antibodies against Spike Protein of SARS-CoV-2. Int. J. Mol. Sci. 21, 9167 (2020). 
bioRxiv preprint doi: https://doi.org/10.1101/2021.05.04.442701; this version posted May 5, 2021. The copyright holder for this preprint (which was not certified by peer review) is the author/funder. All rights reserved. No reuse allowed without permission.

618 82. Amanat, F. et al. An In Vitro Microneutralization Assay for SARS-CoV-2 Serology and Drug Screening. Curr. Protoc. Microbiol. 58, e108 (2020).

83. Siniavin, A. E. et al. Snake venom phospholipases A2 possess a strong virucidal activity against SARS-CoV-2 in vitro and block the cell fusion mediated by spike glycoprotein interaction with the ACE2 receptor. bioRxiv 2021.01.12.426042 (2021). doi:10.1101/2021.01.12.426042 